\title{
Research on Launch Dynamics Modeling and Simulating for Surface-to-air Missile Supported by Adapter
}

\author{
Liu Chaoqi1 ${ }^{1,2, a}$, Wen LiHua ${ }^{1}$, He Jiangjun ${ }^{2}$ and Liu Yuxiang ${ }^{2}$ \\ ${ }^{1}$ Northwestern Polytechnical University, Xi'an 710072, China \\ ${ }^{2}$ Jiangnan Institute of Electromechanical system design, Guiyang 55009, China
}

\begin{abstract}
The launch dynamics analysis model of surface-to-air missile supported by the adapter was established, and the model was verified by test data. Simulation analysis shows: the missile begins to generate pitch angular speed at semi-constrained state after mid-adapter leave the tube, and the speed increases with distance between the mid-adapter and the rear adapter, and as the thrust of the engine increases to decreases, as the launch angle decreases, the missile's pitch angular speed increases, gravity is the main cause of the launch disturbance in the direction of the pitching.
\end{abstract}

\section{Introduction}

The adapter plays a role in fixing, supporting, positioning and launching the missile in the launcher, and has the advantages of simple structure, small size, light weight, and easy loading compared with conventional launch canister with launch rail $[1,2]$. For single vehicle integrated surface-to-air weapon system, reducing the size and weight of the canister can increase the number of missile on the vehicle, or improve the maneuverability of the weapon system, and improve the combat effectiveness. Therefore, the adapter launch technology offers the possibility to design light miniaturization launch canister for surface-to-air system.

When using adapter technology for oblique tracking launch surface-to-air missile, there is a low head disturbance phenomenon during launch compare with those ugs left the rail at the same time, that is the pitch angular speed of the missile gradually increases and the launch angle decreases, it will affect the guidance radar capture the missile or seeker track target, the missile's departure posture is a prerequisite for the reliable operation of the guidance and control system [3]. Therefore, launch dynamics analysis is needed for surface-to-air missile with adapter launch technology, to study the main factors affecting launch disturbance and extract the relationship between launch disturbance and launch angle [4], as design basis for tracking rules of surface-to-air missile launcher and guidance radar interception scheme, etc.

\section{Launch dynamic modeling and model verification}

\subsection{Structure Model and Work Principle}

The missile is supported by 3 sets of adapters in the canister, including the front adapter, the mid-adapter and the rear adapter, the adapter and missile body cooperate with each other through convex block, 4 in each set, as shown in Figure 1(the missile wing is not shown), $\theta$ is the launch angle, and the missile's pitch angle is equal to the launch angle at the beginning. After the solid rocket engine is ignited, the adapter moves along with the missile under the thrust of the engine, the adapter slides along the canister wall, and the front, middle and rear adapters come out in turn, and separates from the missile.

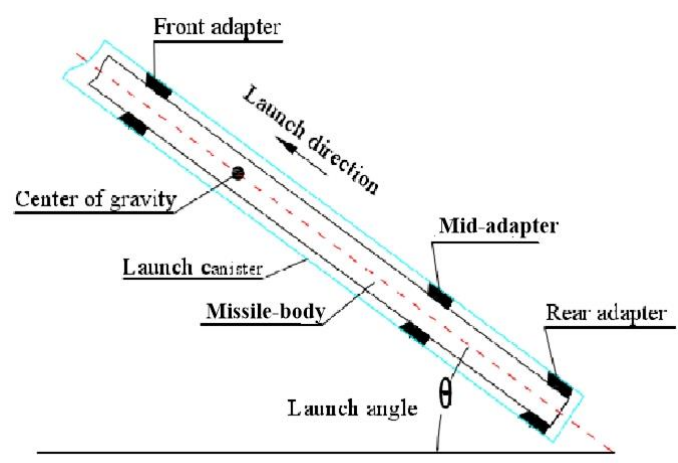

Figure 1. Adapter lateral support position diagram

Each set of adapters is installed in a "X" type, one of which is fitted with the guide bar in the canister, which limits the missile to roll in the canister during the missile launching process, as shown in Figure 2. 


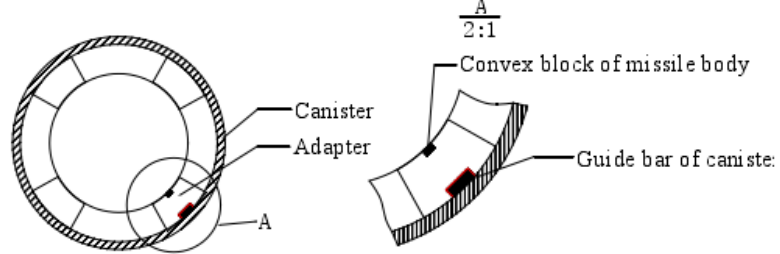

Figure 2.Schematic diagram of adapter circumferential support position

The adapter is composed of adapter body, separating spring, spring press block and so on, as shown in Figure 3. When the adapter is matched with the convex block of the missile body, the block compresses the spring pressure block, and the spring compresses and stores energy. After the adapter is out of the canister, the spring pressure block is unconstrained and the adapter is separated from the missile body under the action of the spring force.
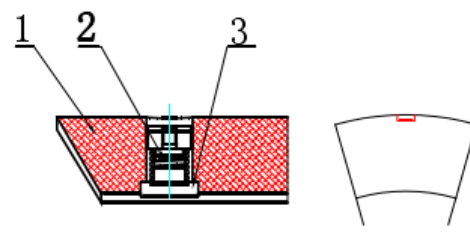

1、Adapter body

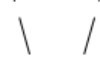

2、 Separating spring

3、 Spring press block

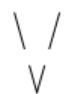

Figure 3. Schematic diagram of adapter structure

\subsection{The suffer force analysis of the missile}

The missile launching process can be divided into three stages: the initial stage, the mid-adapter and the rear adapter in the tube stage and the rear adapter in the tube stage. The force of the missile in these three stages is different and should be analyzed separately.

\subsubsection{The initial stage}

The initial stage missile force is shown in Figure 4. The missile suffers the effects of gravity $G$, engine thrust $P$, friction $\mathrm{f1}$, and adapter support (F11, F12, F13) of the 3 sets of adapters, the missile is in a state of overconstrained. The motion of the missile is relatively stable, but considering the clearance between the missile, the adapters and canister, and the actual structure is elastic, the motion of the missile will have a limited jitter.

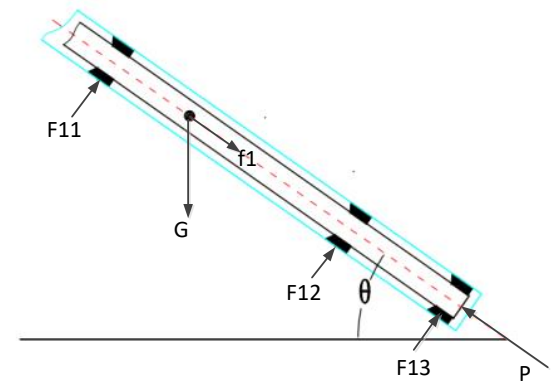

Figure 4. The force during the initial stage

\subsubsection{The mid-adapter and the rear adapter in the tube stage}

When the front adapter left the tube, the missile force is shown in Figure 5. The missile suffers the effects of gravity $\mathrm{G}$, engine thrust $\mathrm{P}$, friction $\mathrm{f} 2$, and adapter support (F22, F23) of the mid-adapters and rear adapters, the missile is in a state of complete constraint state. As the clearance between the missile, the adapters and canister, under the influence of the moment generated by the gravity component, the missile will produce a pitch angular speed, and because the clearance is small, pitch angular speed may oscillate.

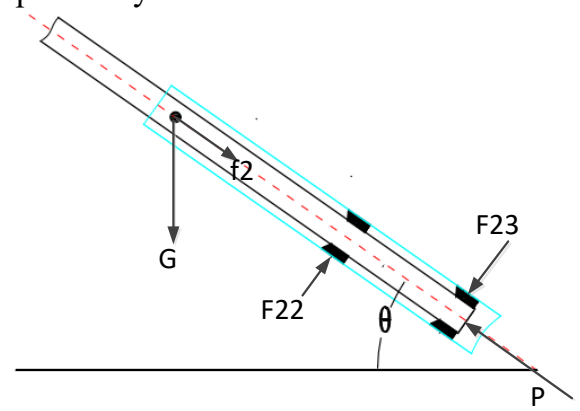

Figure 5. The force during the mid-adapter and the rear adapter in the tube stage

\subsubsection{The rear adapter in the tube stage}

When the mid-adapter left the tube, the missile force is shown in Figure 6. The missile suffers the effects of gravity $G$, engine thrust $P$, friction $\mathrm{f} 3$, and rear adapter support F33, the missile is in an state of semiconstrained state, under the influence of the moment generated by the gravity component, the pitch angular speed will continue increase until the rear adapter left the tube completely.

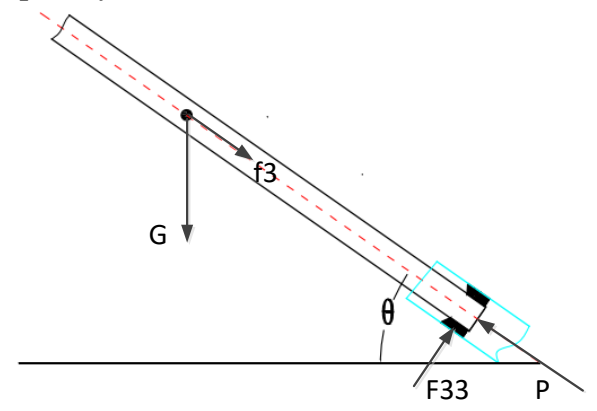

Figure 6. The force during the rear adapter in the tube stage

\subsection{Multi-body dynamic modeling}

Based on ADAMS to build multi-body dynamics model, in order to simplify the model, the following assumptions are made.

(1) The speed of missile during launch is relatively low (less than $25 \mathrm{~m} / \mathrm{s}$ ), aerodynamic force is relatively small, ignoring the influence of aerodynamic forces on missiles and adapters.

(2) Small fitting clearance is used between the missile body, the maximum theoretical gap is $0.5 \mathrm{~mm}$, 
and the stiffness of the missile body and the tube is much greater, can be considered as rigid bodies.

(3) As the constraint of the canister, the launch disturbance caused by the thrust off-center of the engine is suppressed, and engine thrust off-center is ignored.

(4) The impact of the vehicle's elastic vibration is not taken into consideration, and the launch canister is rigidly connected with the earth.

The connection and movement relations among the missile, adapter and canister are shown in Figure 7, and the multi-body dynamics model is built under the those relations.

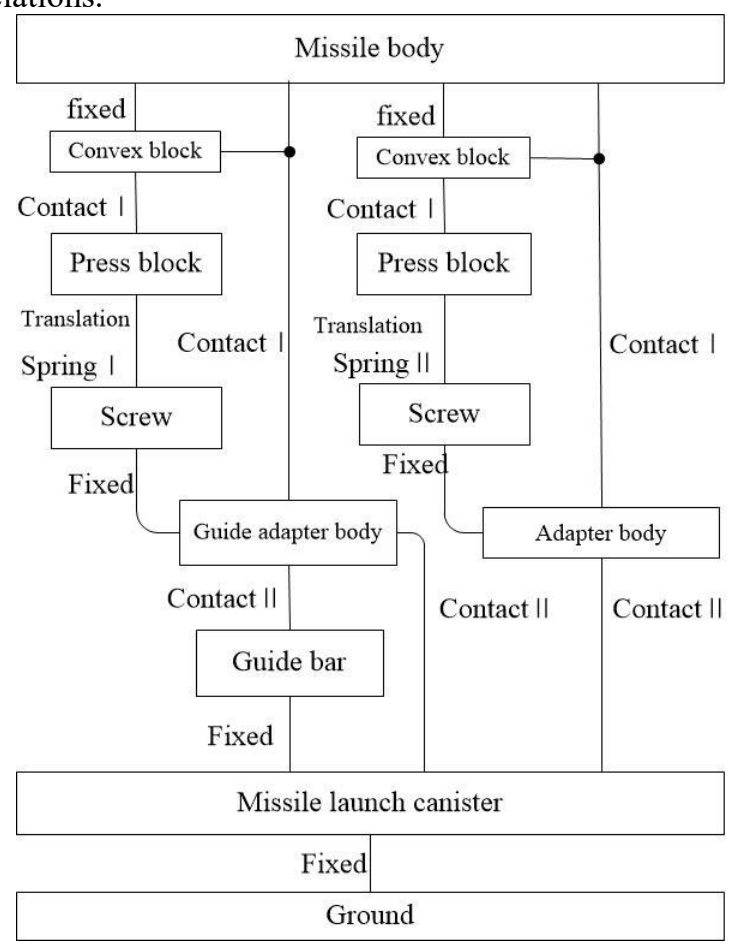

Figure 7. Connection and movement relations of missile, adapters, and launch canister

\subsection{Model verification}

Because the roll and yaw directions are constrained by the tube during missile launch, the roll and yaw disturbances are small. Therefore, the pitch direction disturbance is mainly analyzed.

The simulated calculation of the pitch angel velocity and the data obtained from the launch test are shown in Figure 8, the test data was low-pass filtered. It can be seen that:

(1) The pitch angular speed calculated by the multibody dynamics model is consistent with the trend of the test data, and the error of the pitch angular speed after the missile derailment is small;

(2) Before the front adapter outlet, the missile is in a fully constrained state in the canister, the pitch angular speed changes slightly, but after the front adapter outlet, the pitch angular speed fluctuates slightly due to the small fitting clearance, consistent with the conclusion of the force analysis;

(3) After the middle adapter is out of the canister, the missile is in a semi-constrained state and the pitch speed of the missile begins to increase, after the rear adapter is separated from the canister, the pitch speed of the missile is stable and constant.

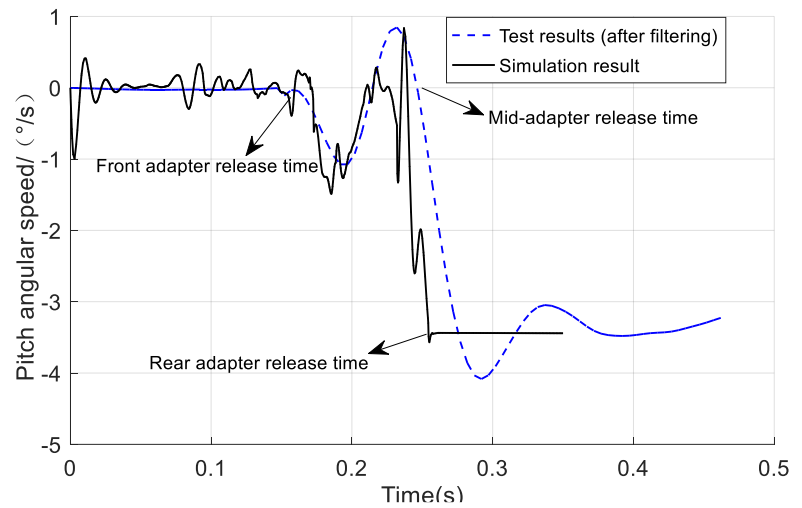

Figure 8. Comparison between simulation results and test data

In conclusion, the multi-body dynamic model has good calculation accuracy, consistent with the physical process, and can be used to carry out the simulation analysis.

\section{Simulation analysis}

As the previous analysis shows that the launch disturbance in the pitch direction of the missile is related to the semi-constrained state and the moment generated by the gravity component, therefore, the adapter support position and launch angle are mainly chosen for analysis.

\subsection{The influence of the adapter support position}

When the mid-adapter leaves the tube, the missile is in an semi-constrained state, and 6 groups of distance working conditions between the mid-adapter and the rear adapter are selected to simulate the launching process of the missile, the launch angle remains unchanged. The calculated mid-adapter release time, missile release time, pitch angular speed are shown in Table 1.

Table 1. Effect of adapter distance between the mid-adapter and the rear adapter on missile launch disturbance

\begin{tabular}{|c|c|c|c|c|}
\hline $\begin{array}{c}\text { No } \\
\cdot\end{array}$ & $\begin{array}{c}\text { Distance } \\
\text { between the } \\
\text { middle adapter } \\
\text { and the rear } \\
\text { adapter(mm) }\end{array}$ & $\begin{array}{c}\text { Medium } \\
\text { adapter } \\
\text { release } \\
\text { time(ms) }\end{array}$ & $\begin{array}{c}\text { Missile } \\
\text { release } \\
\text { time(ms) }\end{array}$ & $\begin{array}{c}\text { Pitch } \\
\text { angular } \\
\text { speed } \\
\left({ }^{\circ} / \mathrm{s}\right)\end{array}$ \\
\hline 1 & 450 & 242 & 258 & -2.42 \\
\hline 2 & 500 & 240 & 259 & -3.03 \\
\hline 3 & 550 & 238 & 259 & -3.44 \\
\hline 4 & 660 & 234 & 259 & -4.11 \\
\hline 5 & 770 & 229 & 260 & -4.78 \\
\hline
\end{tabular}

The following conclusions can be obtained from table 1:

(1) The distance between the middle adapter and the rear adapter does not affect the missile release time, that is, the velocity of the missile is independent from the support position of the adapter; 
(2) The larger the distance between the mid-adapter and the rear adapter, the larger the pitch angular speed, as shown in Figure 9, the smaller the distance between the two is, the more conducive to suppressing the emission disturbance.

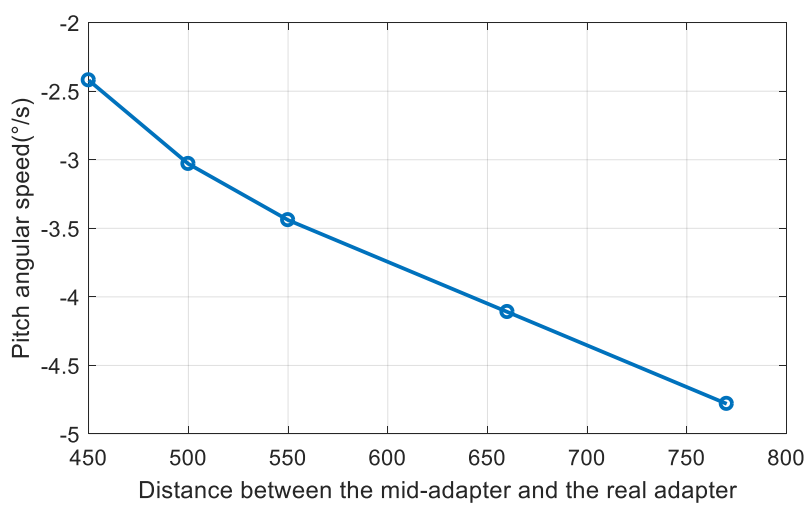

Figure 9. Distance between the mid-adapter and the rear adapter and pitch angular speed relation curve

(3) As the larger the distance between the midadapter and the rear adapter, the longer the missile is in semi-constrained state, the longer the moment of gravity component is applied, the larger the pitch angular speed so, as shown in Figure 10, therefore, the greater the thrust of the engine, the faster the missile will be, and the more it will be beneficial to suppress missile launch disturbances.

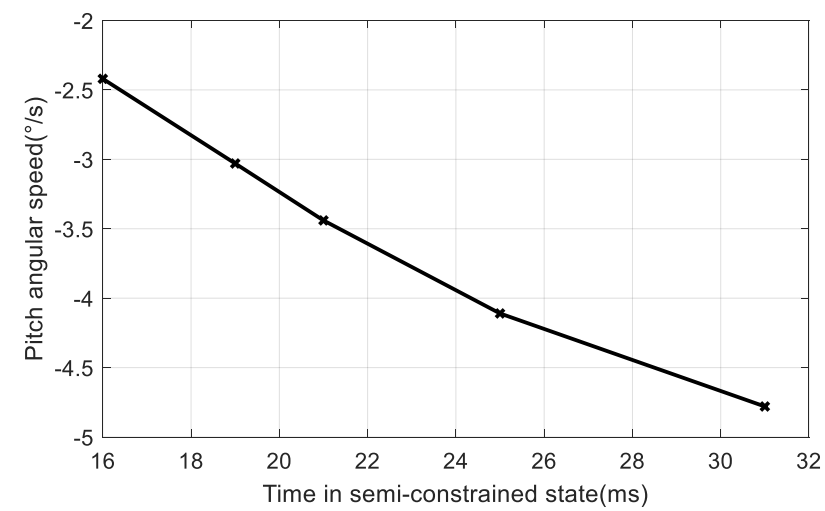

Figure 10. Time in semi-constrained state and pitch angular speed relation curve

In summary, the distance between the mid-adapter and the rear adapter has a great influence on the missile launch disturbance. The smaller the distance between the two is, the more favourable to suppress the launch disturbance. However, the adapter cannot be collided with the expanding airfoil during separation, otherwise, it will be possible to produce serious safety problem [5]. Therefore, the distance between them should be minimized on the premise of guaranteeing the safety of the launch.

\subsection{The influence of launch angle}

The distance between the adapters is kept unchanged, and different launch angles are selected. The calculated parameters and results are shown in Table 2.The following conclusions can be obtained from Table 2:
(1) As the launch angle decreases, the gravity component of the pituitary body increases, the pitching moment increases, and the missile's pitch angular speed increases.

(2) As the launching angle decreases, the time of the missile leaving the canister decreases, indicating that the velocity of the missile's departure from the canister increases, but the pitch angle is still increasing, which indicates that gravity is the main cause of the launch disturbance in the direction of the pitching.

Table 2. Effect of launch angle on missile launch disturbance

\begin{tabular}{|c|c|c|c|c|}
\hline $\begin{array}{c}\text { No } \\
\cdot\end{array}$ & $\begin{array}{c}\text { Launch } \\
\text { angle }^{\circ} \text { ) }\end{array}$ & $\begin{array}{c}\text { Medium } \\
\text { adapter } \\
\text { release } \\
\text { time(ms) }\end{array}$ & $\begin{array}{c}\text { Missile } \\
\text { release } \\
\text { time(ms) }\end{array}$ & $\begin{array}{c}\text { Pitch } \\
\text { angular } \\
\text { speed }\left(^{\circ}\right. \\
/ \text { s) }\end{array}$ \\
\hline 2 & 45 & 225 & 245 & -3.86 \\
\hline 3 & 35 & 223 & 244 & -4.47 \\
\hline 4 & 25 & 222 & 242 & -4.95 \\
\hline 5 & 15 & 220 & 241 & -5.27 \\
\hline
\end{tabular}

\section{Conclusions}

The multi-body dynamic model has good calculation accuracy, which shows the assumptions are reasonable. The following conclusions can be drawn from the results of simulation analysis.

1. When the missile is in a semi-constrained state, it begins to generate pitch angel velocity, reducing the time in semi-constrained state can reduce the launch disturbance.

2. The missile's pitch angular speed increases with the distance between middle adapter and the rear adapter, and as the thrust of the engine increases to decreases.

3. As the launch angle decreases, the missile's pitch angular speed increases, gravity is the main cause of the launch disturbance in the direction of the pitching.

\section{References}

1. C. Meng, T.M. Wang, J.S. WANG. J B UNIV ARERONAUT ASTRONAUT, Research on Geometrical Features of Assembly of Aerocraft and Container, 28, pp.428-431, (2002).

2. H. Zhao, M. Wang, W. Yang, Y.Q. Li, S. Zhao. Tactical Missile technology, Adapters for Canisterlaunched Missile, 4, pp.42-50, (2007).

3. J. Zhao, H.U. Guo, K. Wang, Y. Pan. The study on initial disturbance of the slant-launched missile. Journal of Projectiles Rockets Missiles \& Guidance. 30, pp.76-78, (2010).

4. X. Jiang, P.Y. Liu, W.W. Zhang. Research on typical disturbance factors of anti-aircraft missile initial control point spread effect. Computer Simulation. 32, pp.52-55, (2015). 
5. Y.F Wang, J.G Zhang. Chinese J ASTRONAUT.

Reliability Analysis and calculation of t he missile and Adapter's separating Action, 24, pp.296-300, (2003). 